\title{
Estudo comparativo entre Unidades de Saúde com e sem Estratégia Saúde da Família por meio do PCATool
}

\author{
Comparative Study between Health Care Units with and without Family Health Strategy using \\ PCATool
}

\section{Estudio comparativo de las unidades de salud con y sin la Estrategia Salud de la Familia a través PCATOOI}

Julie Silvia Martins, Silvio Carlos Coelho Abreu, Michele Peixoto Quevedo, Monique Marie Marthe Bourget

\section{Resumo}

Objetivo: Comparar a presença e extensão dos atributos considerados essenciais e derivados da Atenção Primária em Saúde (APS) entre Unidades Básicas de Saúde (UBS) localizadas na zona leste do município de São Paulo que desenvolvem a Estratégia Saúde da Família (ESF) e as UBS tradicionais. Métodos: Todos os médicos (a)s, enfermeiro (a)s e cirurgiões (ãs)-dentistas contratados pela APS Santa Marcelina, que atuam em UBS, foram convidados a participar da presente pesquisa. A coleta de dados foi realizada por meio do PCATool. Resultados: Foi encontrado um melhor desempenho das UBS com ESF em relação aos atributos longitudinalidade $(p=0,0330)$, integralidade (serviços prestados) $(p=0,0011)$, orientação comunitária $(p=0,0017)$ e consequentemente no escore essencial $(0,0327)$ e geral $(0,0098)$, apontando na direção que o processo de trabalho na ESF favorece que tais atributos apresentem melhor desempenho. Conclusão: A Estratégia Saúde da Família tem favorecido agregar qualidade à Atenção Primária à Saúde.

\section{Abstract}

Objective: To compare the presence and extension of attributes that are considered essentials and derived from Primary Health Care (PHC) in Health Care Units (HCU) localized in the eastern region of the city of São Paulo, that work under the optic of Family Health Strategy (FHS) and the ones that do not, called traditional units. Methods: All the physicians, nurses and dentists, hired by the PHC Santa Marcelina institution, who work in these HCU were invited to participate in the study. The data collection was realized by the use of PCATool. Results: The results indicate a better performance of the HCU with FHCS with respect to the PHC attributes such as longitudinality $(p=0.0330)$, integrality (of services offered) $(p=0.0011)$, community orientation $(p=0.0017)$ and consequently in the essential score $(0.0327)$ and in general (0.0098). The scores lead us to infer that the work process in the FHCS offers a better setting for the development of these attributes. Conclusion: It can thus be concluded that HCU with FHCS aggregate more quality to Primary Health Care.

\section{Palavras-chave:} Atenção Primária à Saúde Estratégia Saúde da Família Avaliação de Serviços de Saúde Avaliação em Saúde

\section{Keywords:}

Primary Health Care Family Health Strategy Health Services Evaluation Health Evaluation
Como citar: Martins JS, Abreu SCC, Quevedo MP, Bourget MMM. Estudo comparativo entre Unidades de Saúde com e sem Estratégia Saúde da Família por meio do PCATool. Rev Bras Med Fam Comunidade. 2016;11(38):1-13. http://dx.doi.org/10.5712/rbmfc11(38)1252
Fonte de financiamento: declaram não haver. Parecer CEP: 418/10 (Secretaria Municipal da Saúde - CEP/SMS), aprovado em 07/12/2010.

Conflito de interesses: declaram não haver. Procedência e revisão por pares: revisado por pares. Recebido em: 16/12/2015 Aprovado em: 26/09/2016. 


\section{Resumen}

Objetivo: Comparar la presencia y extensión de los atributos esenciales considerados y derivados de Atención Primaria de Salud (APS) en Unidades Básicas de Salud (UBS), ubicado en la región oriental de Sao Paulo, que desarrollan la Estrategia Salud de la Familia (ESF) y UBS tradicional. Métodos: Todo los médicos(as), enfermeros(as), cirujanos(as)-dentistas contratados por APS Santa Marcelina, que trabajan en UBS fueron invitados a participar en esta investigación. La recolección de datos se realizó a través de PCATool. Resultados: un mejor desempeño se encontró de UBS con FSE en relación a los atributos longitudinalidad ( $p=0,0330)$, exhaustividad (servicios) $(p=0,0011)$, orientación comunitaria $(\mathrm{p}=0,0017)$ y en consecuencia, la puntuación esencial $(0,0327)$ y total $(0,0098)$, que apunta en la dirección de que el proceso de trabajo en la ESF favorece tales atributos presentan un mejor rendimiento. Conclusión: La Estrategia de Salud Familiar ha favorecido la calidad de añadir a la atención primaria de salud.
Palabras clave:

Atención Primaria de Salud

Estrategia de Salud Familiar

Evaluación de

Servicios de Salud

Evaluación en Salud

\section{Introdução}

A Conferência Internacional sobre Cuidados Primários de Saúde em Alma-Ata (1978) ${ }^{1}$ foi um marco dentro da história da saúde, pois foi a primeira vez em que se assumiu mundialmente a saúde como "um direito humano fundamental", propondo que a busca de melhores níveis de saúde fosse considerada "a mais importante meta social mundial" (p. 33). O referido documento também indica que os cuidados primários de saúde constituem o caminho para que esta meta seja atingida.

No Brasil, o direito universal à saúde foi garantido pela Constituição Brasileira em $1988^{2}$ e, desde então, tornou-se o grande desafio a implementação prática da conquista legal.

A Política Nacional de Atenção Básica $(2011)^{3}$ esclarece que a Atenção Primária à Saúde (APS) deve orientar-se pelos princípios da universalidade, acessibilidade, vínculo, continuidade do cuidado, integralidade da atenção, responsabilização, humanização, equidade e participação social.

No cenário internacional, a APS é definida como o primeiro nível de atenção (acesso ao primeiro contato), caracterizando-se, principalmente, pela longitudinalidade, integralidade da atenção e a coordenação da assistência, dentro do próprio sistema de saúde, sendo interessante que também pudesse contar com características complementares como a orientação familiar e comunitária e competência cultural. ${ }^{4-6}$

Mas, até que ponto a APS atende a estes pressupostos?

Shi et al. ${ }^{4}$ desenvolveram um instrumento para avaliar os cuidados recebidos e/ou oferecidos na APS. O Instrumento de Avaliação da Atenção Primária (PCATool - Primary Care Assessment Tool) mede a presença e a extensão dos quatro atributos considerados essenciais (acesso, longitudinalidade, integralidade e coordenação do cuidado) e dos três atributos considerados derivados (orientação familiar, orientação comunitária e competência cultural), possibilitando o cálculo de um escore para cada um dos atributos e para a APS como um todo. A avaliação baseia-se na mensuração de aspectos de estrutura, processos e resultados dos serviços de saúde. ${ }^{7}$ O PCATool permite a comparação do grau de orientação à APS dos diferentes serviços e dos diferentes modelos de atenção básica que coexistem (modelo tradicional e estratégia saúde da família). ${ }^{7}$

De acordo com Harzheim et al., ${ }^{8}$ é fundamental diferenciar a APS da Atenção Mínima à Saúde. Harzheim e seus colaboradores se dedicaram a validar o instrumento para sua utilização no Brasil. ${ }^{9-12}$

Em um momento de grande expansão da Atenção Primária à Saúde no Brasil, principalmente por meio da Estratégia Saúde da Família (ESF), o objetivo do presente trabalho é comparar a presença e extensão 
dos atributos considerados essenciais e derivados da APS em Unidades de Saúde que desenvolvem o modelo tradicional e Unidades de Saúde que desenvolvem a ESF, por meio do instrumento PCATool, considerando inclusive que ambos os modelos estão sendo desenvolvidos dentro da mesma região da cidade, portanto, em contextos parecidos.

\section{Materiais e Métodos}

O método utilizado foi o censo, em que todos os médicos(as), enfermeiros(as) e cirurgiões(ãs)-dentistas contratados pela APS Santa Marcelina que atuavam em Unidades Básicas de Saúde no município de São Paulo foram convidados a participar da pesquisa, independentemente de atuarem ou não na ESF.

Os dados foram coletados por meio do PCATool, em sua versão em português, obtida a partir do Manual publicado pelo Ministério da Saúde. ${ }^{7}$

Para a coleta dos dados, o PCATool foi enviado a cada um dos profissionais, juntamente com o Termo de Consentimento Livre e Esclarecido, explicitando os objetivos da pesquisa e a participação voluntária. Todo o material da pesquisa foi acondicionado em envelope, o qual deveria ser devolvido fechado, para manter o sigilo dos dados. O profissional que não se interessasse em participar da pesquisa poderia apenas colar o envelope e devolver, sem responder o questionário.

Os dados foram digitados e analisados no Programa EPI-INFO versão 3.3.2.

As possíveis respostas para as questões eram: "com certeza sim" (valor=4), "provavelmente sim" (valor=3), "provavelmente não" (valor=2), "com certeza não" (valor=1) e "não sei/não lembro" (valor=9). Os escores dos atributos e de seus componentes foram calculados por meio da média aritmética simples dos valores das respostas. Tais valores foram transformados em escala de 0 a 10 , conforme sugerido no manual do PCATool, ${ }^{7}$ para facilitar o entendimento do leitor e a comparação com resultados de outros estudos. ${ }^{13-16}$

O escore essencial foi calculado a partir da média aritmética dos atributos considerados essenciais (acesso, longitudinalidade, coordenação e integralidade). O escore geral foi calculado a partir da média aritmética de todos os atributos (além dos atributos essenciais, entram no cálculo os escores de orientação familiar e comunitária, que são considerados atributos derivados).

A princípio se pretendia realizar a comparação entre as médias por meio do teste $t$-Student, porém ao testar a suposição de normalidade por meio do teste de Shapiro-Wilk, conforme sugerido por Torman et al., ${ }^{17}$ verificou-se que as variáveis a serem analisadas não apresentavam distribuição "Normal”. Optou-se, então, por utilizar o teste não paramétrico de Kruskal-Wallis para se verificar possíveis diferenças estatisticamente significantes entre as médias dos escores obtidos para as Unidades de Saúde que desenvolvem a ESF e aquelas que desenvolvem o modelo tradicional, considerando a significância estatística quando $p<0,05$.

Como as análises têm o objetivo de buscar possíveis diferenças entre os dois modelos de atenção, optou-se por retirar do banco de dados as Unidades mistas, ou seja, que desenvolvem diferentes modelos de atenção concomitantemente.

Pela extensão do instrumento optou-se em apresentar somente as variáveis com diferença estatisticamente significante entre os dois modelos. Desta forma, as tabelas foram construídas a partir dos atributos, apresentando-se as variáveis referentes àquele atributo com diferença estatisticamente significante. 
A análise foi concluída com a apresentação do escore dos diferentes atributos obtidos nos dois modelos de atenção estudados, bem como o escore derivado e o escore geral, identificando aqueles com diferença estatisticamente significante.

O Projeto de Pesquisa foi aprovado pelo Comitê de Ética em Pesquisas da Secretaria Municipal de Saúde de São Paulo por meio do parecer 418/10 CEP/SMS.

\section{Resultados}

Participaram da pesquisa 317 profissionais, porém, como se optou retirar as Unidades mistas, participaram da presente análise 56 profissionais que atuam em 7 Unidades de Saúde que desenvolvem o modelo tradicional e 243 profissionais que atuam em Unidades Básicas de Saúde que desenvolvem a ESF, envolvendo, portanto, a opinião de 299 profissionais que atuam na APS. Do total de participantes desta análise, 99 (33,1\%) são médico(a)s, 154 (51,5\%) enfermeiro(a)s e 44 (14,7\%) cirurgiões dentistas, e $2(0,7 \%)$ questionários não traziam esta informação.

A maioria dos profissionais participantes - 217 (72,6\%) - era do sexo feminino, 65 (21,7\%) do sexo masculino e 17 (5,7\%) das fichas não apresentavam esta informação. A média de anos que os profissionais trabalham na Unidade Básica de Saúde tradicional é de 5,9 anos e na ESF 5,8, não apresentando diferença estatisticamente significante $(p=0,0761)$.

Em relação ao acesso, os dados apresentados na Tabela 1 demonstram que as Unidades de Saúde tradicionais apresentaram horários de atendimento mais extensos, permanecendo mais frequentemente abertas até as 20 horas quando comparadas às Unidades de Saúde que desenvolvem a ESF $(p=0,0000)$.

Tabela 1. Variáveis referentes à acessibilidade, longitudinalidade e coordenação (integração dos cuidados) que apresentaram diferença estatisticamente significante $(p<0,05)$ entre as médias obtidas para as Unidades de Saúde que seguem o modelo tradicional (UBS) e as Unidades de Saúde que desenvolvem a Estratégia Saúde da Família (ESF). São Paulo, 2011.

\begin{tabular}{|c|c|c|c|}
\hline Acesso de primeiro contato - Acessibilidade & Média ESF & Média UBS & $\mathbf{p}$ \\
\hline A2 - Seu serviço de saúde está aberto, pelo menos em alguns dias da semana até as 20 hs? & 1,76 & 4,53 & 0,0000 \\
\hline \multicolumn{4}{|l|}{ Longitudinalidade } \\
\hline $\begin{array}{l}\text { B1 - No seu serviço de saúde, os pacientes são sempre atendidos pelo mesmo } \\
\text { médico/enfermeiro? }\end{array}$ & 6,65 & 5,77 & 0,0145 \\
\hline B2 - Você consegue entender as perguntas que seus pacientes lhe fazem? & 8,87 & 9,52 & 0,0060 \\
\hline $\begin{array}{l}\text { B7 - Você conhece mais seus pacientes como pessoa do que somente como alguém com um } \\
\text { problema de saúde? }\end{array}$ & 7,44 & 6,13 & 0,0025 \\
\hline B8 - Você sabe quem mora com cada um de seus pacientes? & 5,69 & 2,02 & 0,0000 \\
\hline B11 - Você sabe qual o trabalho ou emprego de cada paciente? & 4,76 & 3,89 & 0,0260 \\
\hline \multicolumn{4}{|l|}{ Coordenação (integração dos cuidados) } \\
\hline $\begin{array}{l}\text { C2 - Quando seus pacientes necessitam um encaminhamento, você discute com os } \\
\text { pacientes sobre diferentes serviços onde eles poderiam ser atendidos? }\end{array}$ & 7,40 & 6,37 & 0,0132 \\
\hline
\end{tabular}

Em relação ao atributo longitudinalidade, entre as variáveis que apresentaram diferenças estatisticamente significantes, as Unidades de Saúde que desenvolvem a ESF tiveram média mais alta para as questões: ser acompanhado (a) pelo mesmo médico (a)/enfermeiro(a), conhecer mais o paciente como pessoa do que somente como alguém com um problema de saúde, saber com quem o paciente mora e qual o trabalho ou emprego do mesmo. 
Quanto à integração dos cuidados, os profissionais que atuam na ESF afirmaram que discutem mais frequentemente com os pacientes sobre os diferentes serviços onde poderiam ser atendidos, de uma forma estatisticamente significante.

Em relação à "coordenação - sistema de informações" nenhuma das variáveis apresentou diferença estatisticamente significante entre o modelo tradicional e as Unidades de Saúde que desenvolvem a ESF, portanto, os dados não foram apresentados.

Quanto à integralidade, que considera os serviços disponíveis, a maioria das variáveis que apresentaram diferença estatisticamente significativa tiveram médias maiores para a ESF, exceto: avaliação de saúde bucal; tratamento dentário e aconselhamento para problemas de saúde mental. Estas apresentaram vantagem para o modelo tradicional de uma forma estatisticamente significante, como pode ser observado na Tabela 2.

Tabela 2. Variáveis referentes à integralidade - serviços disponíveis que apresentaram diferença estatisticamente significante $(p<0,05)$ entre as médias obtidas para as Unidades de Saúde que seguem o modelo tradicional (UBS) e as Unidades de Saúde que desenvolvem a Estratégia Saúde da Família (ESF). São Paulo, 2011.

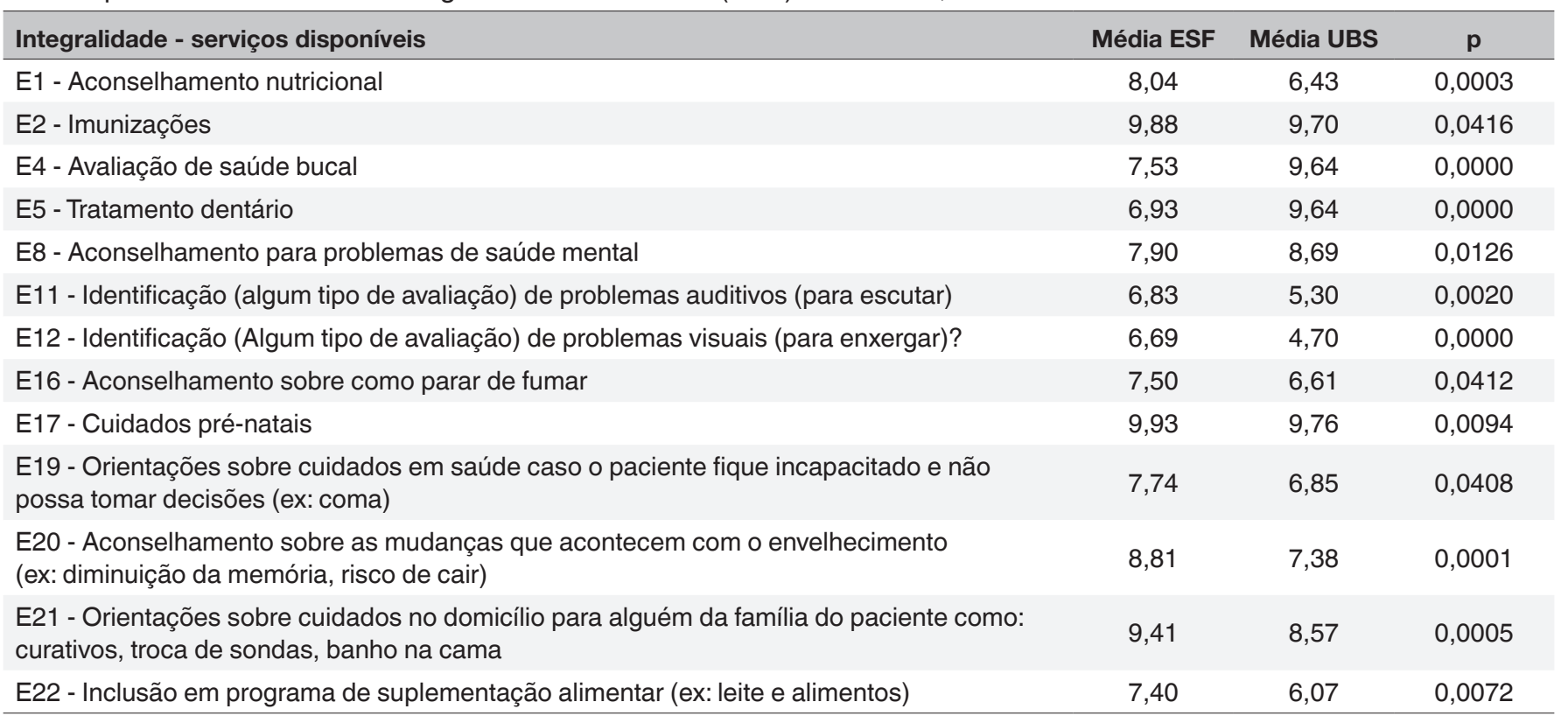

Em relação à integralidade dos serviços prestados, todas as variáveis que apresentaram diferença estatisticamente significativa tiveram melhores médias entre os profissionais que desenvolvem a ESF, como pode ser observado na Tabela 3. A média geral do atributo integralidade (serviços prestados) também se apresentou maior em Unidades que desenvolvem a ESF, de uma forma estatisticamente significante, como pode ser observado na Tabela 5.

Em relação ao atributo "orientação familiar", embora em todas as questões a média das Unidades de Saúde que desenvolvem a ESF fosse mais alta, nenhuma variável apresentou diferença estatisticamente significante e, portanto, os dados não foram apresentados.

Quanto à orientação comunitária (Tabela 4), das seis variáveis estudadas, quatro apresentaram significância estatística a favor da ESF, bem como o atributo como um todo (Tabela 5). 
Tabela 3. Variáveis referentes à integralidade - serviços prestados que apresentaram diferença estatisticamente significante $(\mathrm{p}<0,05)$ entre as médias obtidas para as Unidades de Saúde que seguem o modelo tradicional (UBS) e as Unidades de Saúde que desenvolvem a Estratégia Saúde da Família (ESF). São Paulo, 2011.

\begin{tabular}{|c|c|c|c|}
\hline Integralidade - serviços prestados & Média ESF & Média UBS & $\mathbf{p}$ \\
\hline F1 - Conselhos sobre alimentação saudável ou sobre dormir suficientemente & 9,44 & 8,67 & 0,0029 \\
\hline F2 - Segurança no lar, ex: como guardar medicamentos em segurança. & 8,92 & 7,09 & 0,0000 \\
\hline \multicolumn{4}{|l|}{$\begin{array}{l}\text { Perguntas F3 - F13 se aplicam apenas a adultos (18 anos e acima). "Você discute os } \\
\text { seguintes assuntos com seus pacientes?" }\end{array}$} \\
\hline $\begin{array}{l}\text { F3 - Aconselhamento sobre o uso de cinto de segurança, assentos seguros para crianças } \\
\text { ao andar de carro, evitar que crianças tenham queda de altura }\end{array}$ & 6,94 & 5,25 & 0,0011 \\
\hline F4 - Maneiras de lidar com conflitos de família que podem surgir de vez em quando & 7,89 & 6,88 & 0,0090 \\
\hline F5 - Conselhos a respeito de exercícios físicos apropriados & 8,69 & 8,03 & 0,0322 \\
\hline F6 - Testes de sangue para verificar os níveis de colesterol & 9,15 & 8,26 & 0,0324 \\
\hline F7 - Verificar e discutir os medicamentos que o paciente está usando & 9,45 & 8,91 & 0,0172 \\
\hline F10 - Como prevenir queimaduras causadas por água quente, óleo quente & 7,60 & 5,65 & 0,0005 \\
\hline F11 - Como prevenir quedas & 8,19 & 7,15 & 0,0121 \\
\hline F12 - Prevenção de osteoporose em mulheres & 8,35 & 6,87 & 0,0088 \\
\hline F13 - Cuidado de problemas comuns relativos a menstruação ou a menopausa & 8,61 & 7,45 & 0,0360 \\
\hline \multicolumn{4}{|l|}{$\begin{array}{l}\text { Perguntas F14 - F15 se aplicam apenas a crianças. "Os seguintes assuntos são } \\
\text { discutidos com a criança e pais/responsável?" }\end{array}$} \\
\hline $\begin{array}{l}\text { F15 - Mudanças do crescimento e desenvolvimento da criança esperadas para cada } \\
\text { faixa etária }\end{array}$ & 8,99 & 8,12 & 0,0145 \\
\hline
\end{tabular}

Tabela 4. Variáveis referentes à orientação comunitária que apresentaram diferença estatisticamente significante $(p<0,05)$ entre as médias obtidas para as Unidades de Saúde que seguem o modelo tradicional (UBS) e as Unidades de Saúde que desenvolvem a Estratégia Saúde da Família (ESF), São Paulo, 2011.

\begin{tabular}{|c|c|c|c|}
\hline Orientação comunitária & Média ESF & Média UBS & p \\
\hline H1 - Você ou alguém do seu serviço de saúde faz visitas domiciliares? & 9,96 & 9,31 & 0,0000 \\
\hline $\begin{array}{l}\text { H2 - Você crê que seu serviço de saúde tem conhecimento adequado dos problemas de } \\
\text { saúde da comunidade que atende? }\end{array}$ & 8,78 & 7,05 & 0,0000 \\
\hline $\begin{array}{l}\text { H3 - Seu serviço de saúde ouve opiniões e ideias da comunidade de como melhorar os } \\
\text { serviços de saúde? }\end{array}$ & 8,96 & 8,05 & 0,0004 \\
\hline $\begin{array}{l}\text { H6 - Presença de usuários no Conselho Local de Saúde (Conselho Gestor, Conselho de } \\
\text { Usuários) }\end{array}$ & 9,31 & 8,62 & 0,0027 \\
\hline
\end{tabular}

Tabela 5. Escores médios obtidos para cada um dos atributos da Atenção Primária à Saúde na perspectiva dos profissionais contratados pela APS Santa Marcelina que atuam em Unidades de Saúde que desenvolvem o modelo tradicional (UBS) e em Unidades de Saúde que desenvolvem a Estratégia Saúde da Família (ESF). São Paulo, 2011.

\begin{tabular}{|c|c|c|c|c|}
\hline Dimensão & ESF & UBS & p & Significância estatística \\
\hline Acessibilidade & 3,97 & 3,95 & 0,5885 & \\
\hline Longitudinalidade & 7,00 & 6,56 & 0,0330 & * \\
\hline Coordenação (sistema de informações) & 8,27 & 8,02 & 0,6913 & \\
\hline Integralidade (serviços disponíveis) & 7,35 & 7,12 & 0,0932 & \\
\hline Orientação familiar & 8,50 & 8,08 & 0,1666 & \\
\hline Orientação comunitária & 8,18 & 7,46 & 0,0017 & * \\
\hline Escore essencial & 6,96 & 6,63 & 0,0327 & * \\
\hline Escore geral & 7,31 & 6,89 & 0,0098 & * \\
\hline
\end{tabular}

${ }^{*}$ Apresentou diferença estatisticamente significante $(p<0,05)$. 
Considerando os diferentes atributos da APS abordados no presente estudo, a longitudinalidade, a integralidade (serviços prestados) e a orientação comunitária na perspectiva dos profissionais apresentaram melhor desempenho em Unidades de Saúde que desenvolvem a ESF, de uma forma estatisticamente significante, como pode ser observado na Tabela 5.

O Escore essencial (média aritmética dos escores dos atributos considerados essenciais), bem como o escore geral (média aritmética dos escores de todos os atributos) também apresentaram diferença estatisticamente significante entre os dois modelos estudados, apontando um melhor desempenho das Unidades de Saúde que desenvolvem a ESF.

Os resultados apontam que o atributo "acesso" teve uma média muito baixa e não apresentou diferença estatisticamente significante entre as Unidades de Saúde que desenvolvem o modelo tradicional e aquelas que desenvolvem a ESF, como pode ser observado na Tabela 5.

O acesso foi o único atributo que apresentou média inferior a 6,6, nos dois modelos de atenção.

\section{Discussão}

Embora em outros estudos os questionários do PCATool sejam aplicados por entrevistadores, avaliou-se que tal metodologia poderia inibir o participante a dar a resposta que julgasse mais adequada, por ser necessário compartilhá-la com uma outra pessoa, considerando-se principalmente tratar-se de uma pesquisa institucional. Desta forma, preferiu-se entregar o questionário ao colaborador para que o mesmo pudesse preenchê-lo de acordo com o que julgasse mais adequado, entregando o envelope lacrado, procurando resguardar o sigilo.

Para Macinko e Harzheim ${ }^{12}$ o PCATool, enquanto ferramenta, aponta os pontos fracos, indicando o que e onde há necessidade de melhorias, favorecendo a construção de metas direcionadas às fragilidades encontradas, funcionando como um verdadeiro instrumento de gestão da APS.

Cabe esclarecer que entre os objetivos que nortearam o desenvolvimento da pesquisa também foi a possibilidade de utilizar os resultados na gestão dos serviços envolvidos, uma vez que a análise minuciosa obtida por meio da aplicação do PCATool possibilitou identificar os aspectos que precisariam ser melhorados. Desta forma, foram construídos relatórios com dados agregados por Unidade de Saúde que foram discutidos com as gerências locais, buscando-se aperfeiçoar o modelo.

Os autores ${ }^{13,15,16,18}$ costumam transformar os escores obtidos em valores de (0) zero a 10 (dez), considerando valores inferiores a 6,6 como baixo escore e valores iguais ou superiores a 6,6 como alto escore. Castro et al. ${ }^{16}$ justificam este ponto de corte $(6,6)$ por corresponder, na escala de 1 a 4 , ao escore 3 ("provavelmente sim"). O único atributo que recebeu média inferior a 6,6 nas duas modalidades de assistência básica avaliadas foi o acesso.

A falta de acesso também foi identificada por Macinko et al., ${ }^{19} \mathrm{em}$ Petrópolis; Elias et al., ${ }^{20}$ no município de São Paulo; Chomatas, ${ }^{15}$ em Curitiba; Castro et al., ${ }^{16}$ em Porto Alegre e van Stralen et al., ${ }^{21}$ em Goiás. De acordo com Elias et al., ${ }^{20}$ tais achados provavelmente indicam dificuldades estruturais na oferta de serviços, muito além da organização interna das unidades.

No presente estudo, a única questão relativa ao acesso que apresentou diferença estatisticamente significante entre as duas modalidades avaliadas diz respeito ao serviço estar aberto pelo menos em 
alguns dias da semana até as 20 hs. A diferença foi a favor do modelo tradicional, ficando evidente que resolver esta questão passa necessariamente por mais investimentos financeiros para ampliar o horário de atendimento, favorecendo o acesso aos trabalhadores.

Pode-se considerar o acesso como o atributo prioritário, pois, uma vez que não esteja presente, inviabiliza a consecução dos demais atributos.

A Política Nacional de Atenção Básica, ${ }^{3}$ ao descrever os fundamentos e diretrizes, aponta a necessidade de realizar a adscrição dos usuários, desenvolver a responsabilização entre as equipes e a população adscrita, bem como estabelecer relações de vínculo, garantindo a continuidade das ações de saúde e a longitudinalidade do cuidado.

Entre as variáveis que procuraram acessar o escore do atributo longitudinalidade, quase todas que apresentaram diferença estatisticamente significante entre os dois modelos de atenção apresentaram melhor desempenho na ESF (Tabela 1), indicando que na opinião dos profissionais que a equipe de saúde da família apresenta maior vínculo com o usuário (conhece mais o paciente como pessoa do que somente como alguém com um problema de saúde, sabe com quem o paciente mora e qual o seu trabalho ou emprego). Mas também é preciso se atentar ao fato de que, apesar do melhor desempenho da ESF, as questões com quem o paciente mora e onde é seu trabalho ou emprego apresentaram baixo escore $(<6,6)$ nos dois modelos de atenção.

Macinko et al., ${ }^{19}$ Ibañez et al. ${ }^{22}$ e Chomatas, ${ }^{15}$ ao aplicarem o PCATool, também identificaram diferenças estatisticamente significantes no escore do atributo longitudinalidade entre as Unidades de Saúde tradicionais e as Unidades de Saúde que desenvolvem a ESF.

Uma possível explicação para essas diferenças seria que a organização do processo de trabalho no PSF favorece a longitudinalidade, pois os profissionais que trabalham na ESF têm um contato mais próximo com seus pacientes em decorrência de atendê-lo em consultas subsequentes, discutir os casos em reunião de equipe, ter contato com estes pacientes em grupos e outras atividades promovidas pela Unidade de Saúde, realizar visitas domiciliares, favorecendo, desta forma, o vínculo como ser humano e não apenas na relação profissional médico-paciente.

O escore do atributo longitudinalidade como um todo apresentou um desempenho melhor na ESF, quando comparado ao modelo tradicional (Tabela 5).

Em relação à coordenação (integração dos cuidados), a única variável que apresentou diferença estatisticamente significante entre os dois modelos foi a que procurou verificar se o profissional discute com os pacientes sobre diferentes serviços onde eles poderiam ser atendidos. A média mais alta foi encontrada nas Unidades que desenvolvem a ESF, indicando que estes profissionais dão mais opções de locais de atendimento ao paciente.

Macinko et al. ${ }^{19}$ Cunha, $^{18}$ Gonçalves $^{14}$ e Chomatas $^{15}$ também não encontraram diferença estatisticamente significantes entre os dois modelos de atenção para o atributo coordenação (integração dos cuidados).

Em relação à coordenação do cuidado, um aspecto que apresentou média baixa em ambos os modelos, mas que não foi disponibilizado na tabela, pois não apresentou diferença estatisticamente significante entre os modelos estudados, refere-se à informação recebida do especialista a respeito do paciente encaminhado (contrarreferência). 
A média apontada pelos profissionais que desenvolvem a ESF foi 3,33 e daqueles que trabalham em UBS foi de 4,23, demonstrando nítida insatisfação dos profissionais em relação às informações recebidas da contrarreferência, indicando a necessidade da gestão trabalhar a questão das relações interinstitucionais, estabelecendo protocolos que possibilitem dar mais informações aos profissionais que atuam na Atenção Básica a respeito de seus pacientes, para que os mesmos possam dar continuidade ao atendimento deste paciente.

No atributo coordenação (sistema de informação), nenhuma questão apresentou diferença estatisticamente significante entre os dois modelos de atenção, situação esta encontrada por outros autores. ${ }^{15,16,18,19,22}$

Em relação ao atributo integralidade - serviços disponíveis, verificou-se diferença estatisticamente significante em vários aspectos, como pode ser observado na Tabela 2. O que se observa é que as Unidades de Saúde tradicionais apresentam vantagem em relação à saúde bucal (avaliação e tratamento dentário) e aconselhamento para problemas de saúde mental.

As Unidades de Saúde que desenvolvem a ESF apresentaram melhor desempenho nos itens: aconselhamento nutricional; imunização; aconselhamento sobre as mudanças que acontecem com o envelhecimento (ex: diminuição da memória, risco de cair); orientações sobre cuidados no domicílio para alguém da família do paciente como: curativos, troca de sondas, banho na cama; orientações caso o paciente fique incapacitado e não possa tomar decisões; aconselhamento sobre como parar de fumar; cuidados pré-natais.

Também se destaca na identificação de problemas visuais e auditivos e inclusão em programas de suplementação alimentar, como pode ser observado na Tabela 2. Tais resultados apontam um desempenho melhor da ESF em práticas de promoção da saúde e prevenção de doenças.

Em relação à saúde bucal, dados do Datasus ${ }^{23}$ indicam que, em março de 2011, o município de São Paulo contava com 1.308 cirugiões-dentistas clínicos gerais atuando em Unidades de Saúde tradicionais e 213 cirurgião-dentista atuando na estratégia de saúde da família. Então, é esperado que uma maior proporção de Unidades de Saúde tradicionais desenvolvessem ações de saúde bucal do que aquelas que desenvolvem a ESF.

Em relação aos dados apresentados na Tabela 2, referentes ao atributo "integralidade - serviços disponíveis", sobre orientações e aconselhamentos, com exceção do aconselhamento para problemas de saúde mental, todos os demais apresentaram vantagem para a ESF quando comparados à Unidade de Saúde tradicional, de uma forma estatisticamente significante.

O mesmo pode ser observado na Tabela 3, na qual são apresentados os dados referentes à "integralidade - serviços prestados", em que as variáveis pesquisadas relacionam-se de uma forma marcante às ações de orientação e prevenção; em todos os casos que se observou uma diferença estatisticamente significante, a vantagem se apresentou relacionada à ESF.

Os achados das Tabelas 2 e 3 parecem indicar que a ESF favorece o desenvolvimento de ações educativas e preventivas, corroborando com os achados de Harzheim \& Álvarez-Dardet ${ }^{24}$ e Gonçalves. ${ }^{14}$

O atributo integralidade - "serviços disponíveis" não apresentou diferença estatisticamente significante entre os dois modelos estudados (Tabela 5), resultado este também encontrado por outros autores, ${ }^{16,18,19}$ 
porém, o atributo integralidade - "serviços prestados" apresentou um desempenho melhor nas Unidades de Saúde que desenvolvem a ESF, concordante ao encontrado por outros autores. ${ }^{15,16,22}$

Conill, ${ }^{25}$ utilizando metodologia qualitativa, procurou avaliar a APS a partir do Programa Saúde da Família em Florianópolis. A autora também observou dificuldades no acesso e efeitos positivos em relação à integralidade da atenção, corroborando com os achados da presente pesquisa.

Em relação ao atributo "orientação comunitária", quatro variáveis apresentaram diferença estatisticamente significante entre os modelos de atenção, com vantagem para a ESF. Tais variáveis versavam sobre a realização de visitas domiciliares, o conhecimento adequado dos problemas de saúde da comunidade, se o serviço de saúde ouve as opiniões e ideias da comunidade de como melhorar os serviços de saúde e a participação popular no Conselho Local de Saúde.

O atributo "orientação comunitária" apresentou um desempenho como um todo melhor na ESF de uma forma estatisticamente significante, achado este que corrobora com o achado de outros autores. ${ }^{14-16,18,21,22,24}$ De uma maneira geral, este resultado já era o esperado, tendo em vista que todo o processo de trabalho da ESF é voltado para que a Equipe estabeleça relação/vínculo com a comunidade atendida, considerando a presença dos Agentes Comunitários de Saúde, o cadastro, o processo de territorialização, o Sistema de Informação, o estímulo à participação social, entre outros.

O que chama a atenção, no sentido positivo, é o fato da UBS tradicional apresentar uma média 9,31 para a pergunta: "Você ou alguém do seu serviço de saúde faz visitas domiciliares?" apontando que as Unidades de Saúde tradicionais estão caminhando nesta direção, ou seja, oferecendo cuidados domiciliares e se aproximando de uma característica das Unidades com ESF, trazendo, é claro, inúmeros benefícios aos cidadãos.

Os escores dos diferentes atributos, exceto o acesso, se apresentaram maiores ou iguais a 6,6, tanto em Unidades que desenvolvem a ESF como nas Unidades de Saúde tradicionais, conforme pode ser observado na Tabela 5. Escores maiores ou iguais a 6,6, conforme apontado anteriormente, são classificados por diferentes autores como alto escore, significando que tais atributos encontram-se presentes em extensão adequada. Sob este ponto de vista, a situação se apresenta bastante favorável aos serviços analisados, mas é preciso considerar, de acordo com Elias et al., ${ }^{20}$ que a percepção dos profissionais é sempre melhor que a dos usuários, fato constatado também por van Stralen et al. ${ }^{21}$

Os atributos longitudinalidade, integralidade (serviços prestados) e a orientação comunitária apresentaram melhor desempenho na ESF (Tabela 5), o que permitiu que a ESF apresentasse um desempenho melhor no escore essencial, achado este concordante ao encontrado por outros autores ${ }^{15,16}$ e também no escore geral, indicando uma maior consolidação dos atributos da APS na Estratégia Saúde da Família, corroborando com os achados de outros autores. ${ }^{15,16,18,24}$ Facchini et al., ${ }^{26}$ utilizando outra metodologia, também encontraram melhores resultados nas Unidades de Saúde que desenvolvem a Estratégia Saúde da Família.

Segundo Macinko et al., ${ }^{19}$ o PSF se apresenta melhor do que as Unidades de Saúde tradicionais em alguns aspectos, mas não de forma generalizada em todas as dimensões dos cuidados primários e foi exatamente o que se constatou na presente pesquisa. 
O fato de ambos os modelos estarem sendo desenvolvidos na mesma região do município e também estarem sob a responsabilidade da mesma parceira da prefeitura (APS Santa Marcelina) favoreceu o desenvolvimento da pesquisa e de possíveis comparações entre os modelos estudados.

Considera-se como uma limitação do presente estudo envolver apenas a opinião dos profissionais, indicando-se a realização de novos estudos nos quais seja possível obter a opinião dos usuários.

Recomenda-se a realização de estudos que possam avaliar a relação entre a presença e extensão dos atributos da APS e níveis de saúde das respectivas comunidades, conforme sugerido por Harzheim et al., ${ }^{8}$ para que se possa aprofundar o conhecimento sobre esta relação e suas consequências.

\section{Considerações finais}

O presente estudo apontou um bom desempenho tanto dos atributos considerados essenciais como dos atributos considerados derivados da APS (escore $\geq 6,6$ ), medidos por meio do PCATool, em ambos os modelos de atenção/assistência, com exceção do acesso, que não apresentou bom desempenho em nenhum dos modelos.

Tais achados apontam que a APS Santa Marcelina, na região, vem sendo construída atendendo aos pressupostos considerados essenciais, bem como aos derivados, necessitando porém de maiores investimentos para garantir o acesso à toda a população, principalmente aos trabalhadores, oferecendo acesso aos mesmos em horários diferenciados.

O melhor desempenho da ESF nos atributos longitudinalidade, integralidade (serviços prestados) e orientação comunitária e, consequentemente, no escore geral, aponta na direção de que o processo de trabalho na ESF favorece que tais atributos apresentem melhor desempenho, concluindo-se, portanto, que a Estratégia Saúde da Família tem favorecido agregar qualidade à Atenção Primária à Saúde.

\section{Referências}

1. Declaração de Alma-Ata. Conferência Internacional sobre cuidados primários de saúde; 6-12 de setembro 1978; Alma-Ata; USSR. In: Ministério da Saúde. Secretaria de Políticas de Saúde. Projeto Promoção da Saúde. As Cartas da Promoção da Saúde. Brasília: Ministério da Saúde; 2002. (Série B. Textos Básicos em Saúde). [acesso 2015 Ago 14]. Disponível em: http://bvsms.saude.gov.br/bvs/publicacoes/cartas_promocao.pdf

2. Brasil. Constituição da República Federativa do Brasil. São Paulo: Rideel; 1999.

3. Brasil. Ministério da Saúde. Portaria nํ⒉488, de 21 de outubro de 2011. Aprova a Política Nacional de Atenção Básica, estabelecendo a revisão de diretrizes e normas para a organização da atenção básica, para a Estratégia Saúde da Família (ESF) e o Programa de Agentes Comunitários de Saúde (PACS). Diário Oficial União; 2011.

4. Shi L, Starfield B, Xu J. Validating the Adult Primary Care Assessment Tool. J Fam Pract. 2001;50(2):161-75.

5. Starfield B. Atenção primária: equilíbrio entre necessidades de saúde, serviços e tecnologias. Brasília: UNESCO, Ministério da Saúde; 2004.

6. Starfield B, Shi L. Manual for the primary care assessment tools. Baltimore: Johns Hopkins University; 2009. [acesso 2015 Set 25]. Disponível em: http://www.jhsph.edu/research/centers-and-institutes/johns-hopkins-primary-carepolicy-center/pca_tools.html

7. Brasil. Ministério da Saúde. Secretaria de Atenção em Saúde. Departamento de Atenção Básica. Manual do instrumento de avaliação da atenção primária à saúde: Primary Care Assessment Tool PCATool. Brasília: Ministério da Saúde; 2010.

8. Harzheim E, Stein AT, Álvarez-Dardet C. A efetividade dos atributos da atenção primária sobre a saúde infantil. Bol Saúde. 2004;18(1):23-40. 
9. Harzheim E, Stein AT, Álvarez-Dardet C, Cantero MTR, Kruse CK, Vidal TB, et al. Revisão sistemáticas sobre aspectos metodológicos das pesquisas em atenção primária no Brasil. Rev AMRIGS. 2005;49(4):248-52.

10. Harzheim E, Starfield B, Rajmil L, Álvarez-Dardet C, Stein AT. Consistência interna e confiabilidade da versão em português do Instrumento de Avaliação da Atenção Primária (PCATool-Brasil) para serviços de saúde infantil. Cad Saúde Pública. 2006; 22(8):1649-59. http://dx.doi.org/10.1590/S0102-311X2006000800013. PMid:16832536

11. Harzheim E, Duncan BB, Stein AT, Cunha CR, Gonçalves MR, Trindade TG, et al. Quality and effectiveness of different approaches to primary care delivery in Brazil. BMC Health Serv Res. 2006;6:156.

12. Macinko J, Harzheim E. Instrumento de Avaliação da Atenção Primária - sua utilização no Brasil - Entrevista. Rev Bras Saúde Fam. 2007;14:4-7.

13. Oliveira MMC. Presença e extensão dos atributos da atenção primária à saúde entre os serviços de atenção primária em Porto Alegre: uma análise agregada [Dissertação de mestrado]. Porto Alegre: Faculdade de Medicina da Universidade Federal do Rio Grande do Sul; 2007.

14. Gonçalves MR. Associação entre a qualidade da atenção primária à saúde e o processo de atenção aos portadores de diabetes mellitus adscritos aos serviços de saúde em Porto Alegre. [Dissertação de mestrado]. Porto Alegre: Faculdade de Medicina da Universidade Federal do Rio Grande do Sul; 2007.

15. Chomatas ERV. Avaliação da presença e extensão dos atributos da atenção primária na rede básica de saúde no município de Curitiba, no ano de 2008 [Dissertação de mestrado]. Porto Alegre: Faculdade de Medicina da Universidade Federal do Rio Grande do Sul; 2009.

16. Castro RCL, Knauth DR, Harzheim E, Hauser L, Ducan BB. Avaliação da qualidade da atenção primária pelos profissionais de saúde: comparação entre diferentes tipos de serviços. Cad Saúde Pública. 2012;28(9):1772-84. http://dx.doi.org/10.1590/S0102-311X2012000900015.

17. Torman VBL, Coster R, Riboldi J. Normalidade de variáveis: métodos de verificação e comparação de alguns testes não-paramétricos por simulação. Rev HCPA. 2012;32(2):227-34.

18. Cunha $\mathrm{CRH}$. Percepção da qualidade da atenção à saúde infantil pelos médicos e enfermeiros: comparação entre o programa saúde da família e o modelo tradicional [Dissertação de mestrado]. Porto Alegre: Faculdade de Medicina da Universidade Federal do Rio Grande do Sul; 2006.

19. Macinko J, Almeida C, dos SE, de Sá PK. Organization and delivery of primary health care services in Petrópolis, Brazil. Int J Health Plann Manage. 2004;19(4):303-17. http://dx.doi.org/10.1002/hpm.766. PMid:15688875

20. Elias PE, Ferreira CW, Alves MCG, Cohn A, Kishima V, Escrivão Junior A, et al. Atenção Básica em Saúde: comparação entre PSF e UBS por estrato de exclusão social no município de São Paulo. Ciênc Saúde Coletiva. 2006;11(3):633-41. http://dx.doi.org/10.1590/S1413-81232006000300012

21. van Stralen CJ, Belisário SA, van Stralen TBS, Lima AMD, Massote AW, Oliveira CL. Percepção dos usuários e profissionais de saúde sobre atenção básica: comparação entre unidades com e sem saúde da família na Região Centro-Oeste do Brasil. Cad Saúde Pública. 2008;24(suppl.1):148-58. http://dx.doi.org/10.1590/S0102-311X2008001300019

22. Ibañez N, Rocha JSY, Castro PC, Ribeiro MCSA, Forster AC, Novaes MHD, et al. Avaliação do desempenho da atenção básica no Estado de São Paulo. Ciênc Saúde Coletiva. 2006;11(3):683-703. http://dx.doi.org/10.1590/S141381232006000300016

23. Brasil. Ministério da Saúde. Banco de dados do Sistema Único de Saúde-DATASUS, Cadastro Nacional de Estabelecimentos de Saúde - CNES Recursos Humanos - profissionais - indivíduos - segundo CBO 2002 - São Paulo. [Acesso 2015 Ago 7]. Disponível em: http://tabnet.datasus.gov.br/cgi/tabcgi.exe?cnes/cnv/prid02sp.def

24. Harzheim E, Álvarez-Dardet C. Evaluación de la atención a la salud infantil de Programa Saúde da Família em la región sur de Porto Alegre, Brasil. Rev Bras Med Fam Comunidade. 2007;3(9):60-1.

25. Conill EM. Políticas de atenção primária e reformas sanitárias: discutindo a avaliação a partir da análise do Programa Saúde da Família em Florianópolis, Santa Catarina, Brasil, 1994-2000. Cad Saúde Pública. 2002;18(suppl.):191-202. http://dx.doi.org/10.1590/S0102-311X2002000700019

26. Facchini LA, Piccini RX, Tomasi E, Thumé E, Silveira DS, Siqueira FV, et al. Desempenho do PSF no Sul e no Nordeste do Brasil: avaliação institucional e epidemiológica da Atenção Básica à Saúde. Ciênc Saúde Coletiva. 2006;11(3):669-81. http://dx.doi.org/10.1590/S1413-81232006000300015 
Julie Silvia Martins. Atenção Primária à Saúde Santa Marcelina.

São Paulo, SP, Brasil. julie@aps.santamarcelina.org

(Autora correspondente)

Silvio Carlos Coelho Abreu. Atenção Primária à Saúde Santa Marcelina. São Paulo, SP, Brasil. silvioabreu@aps.santamarcelina.org.br

Michele Peixoto Quevedo. Atenção Primária à Saúde Santa Marcelina. São Paulo, SP, Brasil. michele @aps.santamarcelina.org

Monique Marie Marthe Bourget. Atenção Primária à Saúde Santa Marcelina.

São Paulo, SP, Brasil. monique @ santamarcelina.org 